\title{
Clinically applicable delineation of the pallidal sensorimotor region in patients with advanced Parkinson's disease: study of probabilistic and deterministic tractography
}

\author{
Jennifer Muller, MS,, Mahdi Alizadeh, PhD, ${ }^{1,2}$ Feroze B. Mohamed, PhD, ${ }^{1}$ Jonathan Riley, MD, ${ }^{2}$ \\ John J. Pearce, MD, ${ }^{3}$ Benjamin Trieu, ${ }^{4}$ Tsao-Wei Liang, MD, ${ }^{5}$ Victor Romo, MD, ${ }^{6}$ \\ Ashwini Sharan, MD, ${ }^{2}$ and Chengyuan $\mathrm{Wu}, \mathrm{MD}^{1,2}$
}

Departments of ${ }^{1}$ Radiology, ${ }^{2}$ Neurosurgery, ${ }^{5}$ Neurology, and ${ }^{6}$ Anesthesiology, Thomas Jefferson University; and ${ }^{3}$ Lewis Katz School of Medicine and ${ }^{4}$ College of Liberal Arts, Temple University, Philadelphia, Pennsylvania

\begin{abstract}
OBJECTIVE Deep brain stimulation (DBS) is an effective procedure in improving motor symptoms for patients with advanced Parkinson's disease (PD) through the use of high-frequency stimulation. Although one of the most commonly used target sites for DBS, sensorimotor regions of the globus pallidus interna (GPi) have yet to be thoroughly described with advanced neuroimaging analysis in vivo for human subjects. Furthermore, many imaging studies to date have been performed in a research setting and bring into question the feasibility of their applications in a clinical setting, such as for surgical planning. This study compares two different tractography methods applied to clinically feasible acquisition sequences in identifying sensorimotor regions of the GPi and the subthalamic nucleus (STN) in patients with advanced PD selected to undergo DBS.
\end{abstract}

METHODS Seven patients with refractory PD selected for DBS were examined by MRI. Diffusion images were acquired with an average acquisition time of 15 minutes. Probabilistic and deterministic tractography methods were applied to each diffusion-weighted data set using FSL and MRtrix, respectively. Fiber assignment was performed using combined sensorimotor areas as initiation seeds and the STN and GPi, separately, as inclusion masks. Corticospinal tracts were excluded by setting the cerebral peduncles as exclusion masks. Variability between proposed techniques was shown using center of gravity (CoG) coordinates.

RESULTS Deterministic and probabilistic corticopallidal and corticosubthalamic pathways were successfully reconstructed for all subjects across all target sites (bilaterally). Both techniques displayed large connections between the sensorimotor cortex with the posterolateral aspect of the ipsilateral GPi and the posterosuperolateral aspect of the ipsilateral STN. The average variability was $2.67 \mathrm{~mm}$, with the probabilistic method identifying the CoG consistently more posterior and more lateral than the deterministic method.

CONCLUSIONS Successful delineation of the sensorimotor regions in both the GPi and STN is achievable within a clinically reasonable timeframe. The techniques described in this paper may enhance presurgical planning with increased accuracy and improvement of patient outcomes in patients undergoing DBS. The variability found between tracking techniques warrants the use of the probabilistic tractography method over the deterministic method for presurgical planning. Probabilistic tractography was found to have an advantage over deterministic tractography in its sensitivity, in accurately describing previously described tracts, and in its ability to detect a larger number of fibers.

https://thejns.org/doi/abs/10.3171/2018.7.JNS18541

KEYWORDS diffusion-weighted imaging; deep brain stimulation; sensorimotor regions of the globus pallidus interna; probabilistic tractography; fronto-striato-subthalamic-pallidal network; functional neurosurgery

\footnotetext{
ABBREVIATIONS COG = center of gravity; CSD = constrained spherical deconvolution; $\mathrm{DBS}=$ deep brain stimulation; DTI = diffusion tensor imaging; FDT = FMRIB Diffusion Toolbox; FMRIB = Functional Magnetic Resonance Imaging of the Brain; FOD = fiber orientation distribution; GPi = globus pallidus interna; GPU = graphics processing unit; MPRAGE = magnetization-prepared rapid acquisition gradient echo; PD = Parkinson's disease; ROI = region of interest; STN = subthalamic nucleus; UPDRS = Unified Parkinson's Disease Rating Scale.
}

SUBMITTED February 23, 2018. ACCEPTED July 16, 2018.

INCLUDE WHEN CITING Published online December 14, 2018; DOI: 10.3171/2018.7.JNS18541. 
$\mathrm{D}$ EEP brain stimulation (DBS) involves the implantation of electrodes that apply high-frequency electrical stimulation to targeted subcortical structures. Over the past 2 decades, DBS has been a well-established treatment option for patients suffering from movement disorders. ${ }^{16}$ Stimulation of the subthalamic nucleus (STN) or globus pallidus pars interna (GPi) has provided significant improvement in motor function in patients with Parkinson's disease (PD) by reducing dyskinesia and motor fluctuations in tremor, rigidity, and bradykinesia. ${ }^{9,23}$

Follett et al. compared 24-month outcomes for patients who had undergone bilateral stimulation of the GPi and STN and found that patients with PD had similar improvements in motor function after either pallidal or subthalamic stimulation. ${ }^{14}$ Stimulation of the GPi has been found to reduce medication side effects, leading to better drug tolerance. ${ }^{24}$ Furthermore, available data comparing cognitive performances following DBS suggest that risk of cognitive decline is lower in GPi DBS when compared to STN. ${ }^{17,27,29}$ Despite the lack of evidence that neurostimulation of the STN provides better outcomes, ${ }^{8}$ this nucleus is more commonly targeted for DBS. ${ }^{14,41}$ Imaging research has followed suit, with the majority of the literature published focusing on the connections involving the STN. ${ }^{22}$ Patriat et al. were able to parcellate the functional territories of the GPi into motor, associative, and limbic regions using tractography-based parcellation. The authors claimed that "the consistency, validity, and clinical relevance of our findings have the potential for improving DBS targeting, by increasing patient-specific knowledge of subregions of the GPi to be targeted or avoided, at the stage of surgical planning, and later, at the stage when stimulation is adjusted." 31 Because individualized treatment planning based on identification of sensorimotor regions of the nuclei has the potential to improve outcomes in patients with DBS, ${ }^{40}$ there remains a need for the identification and delineation of pallidal pathways, specifically the sensorimotor regions of the GPi. Unfortunately, our current understanding of the somatotopic organization of the GPi in humans is limited and a more detailed map of the individual regions of the GPi is desirable for understanding both the effects of DBS and clinical outcomes. . $^{5,35,36}$

Diffusion imaging is a commonly used MRI method to noninvasively trace white matter tracts and map structural connections of the human brain. ${ }^{1}$ Diffusion-sensitized imaging utilizes two magnetic gradients as a means of visualizing the microstructural organization of white matter. ${ }^{26}$ Diffusion-based tractography has become a useful tool to better visualize and understand activated pathways associated with PD. ${ }^{15}$ Algorithms have been developed to generate 3D representations of axonal fibers based on the direction of the major eigenvector of diffusion commonly known as tractography. ${ }^{19}$ Tractography methods use local diffusion orientations to determine pathways between distinct brain regions using two different diffusion model fittings. In general, tractography methods can be classified into deterministic and probabilistic categories. Deterministic tractography assumes a single fiber orientation for each voxel, producing one streamline per seed voxel. Probabilistic tractography, however, assumes a distribution of fiber orientations, and produces multiple stream- line samples per seed voxel drawn from a probability distribution of the orientation estimates (Fig. 1). ${ }^{11}$ Although deterministic methods of diffusion tensor imaging (DTI) are more commonly used to visualize tracts for clinical purposes, a major limitation of deterministic tracking is in its inability to accurately represent crossing fibers, kissing fibers, and fanning fibers in image voxels containing more than one fiber orientation, which have been estimated to comprise $90 \%$ of all voxels. ${ }^{21}$ More advanced diffusion models, such as the constrained spherical deconvolution (CSD) method, may more accurately track voxels containing multiple fiber orientations. Advanced tracking methods provide more anatomically plausible tracts, and may provide more valuable information for neurosurgical planning. ${ }^{6}$ This technique is not commonly used in a clinical setting, as the computations needed for probabilistic tractography are generally much more demanding than deterministic tractography algorithms. ${ }^{11}$ Recent studies, however, have compared tensor-based deterministic methods to advanced probabilistic methods in patients selected for STN DBS and have shown that the CSD-based probabilistic method provides more consistent tractography results when compared to deterministic-based methods. ${ }^{32,38}$ At the same time, these studies were limited by creating target maps only in the region of the $\mathrm{STN}^{32}$ and failed to implement scan times with a duration appropriate for a clinical setting. It has been suggested that fiber pathways reconstructed with CSD are more consistent with known neuroanatomy than those reconstructed with deterministic models, and may better connect pathways in the complex fiber architecture of white matter. ${ }^{25}$

This study evaluates the use of both deterministic and probabilistic tractography methods to delineate three pathways involved in the mediation of DBS treatment effects: the hyperdirect, direct, and indirect pathways. This is the first study to reconstruct not only cortico-STN hyperdirect pathways, but also corticopallidal pathways, using a clinically feasible processing pipeline as well as clinically feasible scan times.

\section{Methods \\ Participants}

In this retrospective single-center study, a total of 7 patients with PD (3 men and 4 women, ranging in age from 69 to 78 years old) selected for DBS were scanned prior to surgery. The study was approved by the IRB of Thomas Jefferson University. All patients had a diagnosis of advanced idiopathic PD according to standard clinical criteria. All patients underwent motor examinations (Unified Parkinson's Disease Rating Scale [UPDRS]-III) before and after image-guided, image-verified DBS under general anesthesia without microelectrode recordings. Based on clinical protocols, 2 patients underwent DBS implantation into the bilateral STN while the remaining 5 patients underwent placement of DBS neurostimulators into the bilateral GPi. Detailed patient characteristics for this main study are presented in Table 1.

\section{Image Acquisition}

All DBS candidates underwent preoperative MRI ex- 


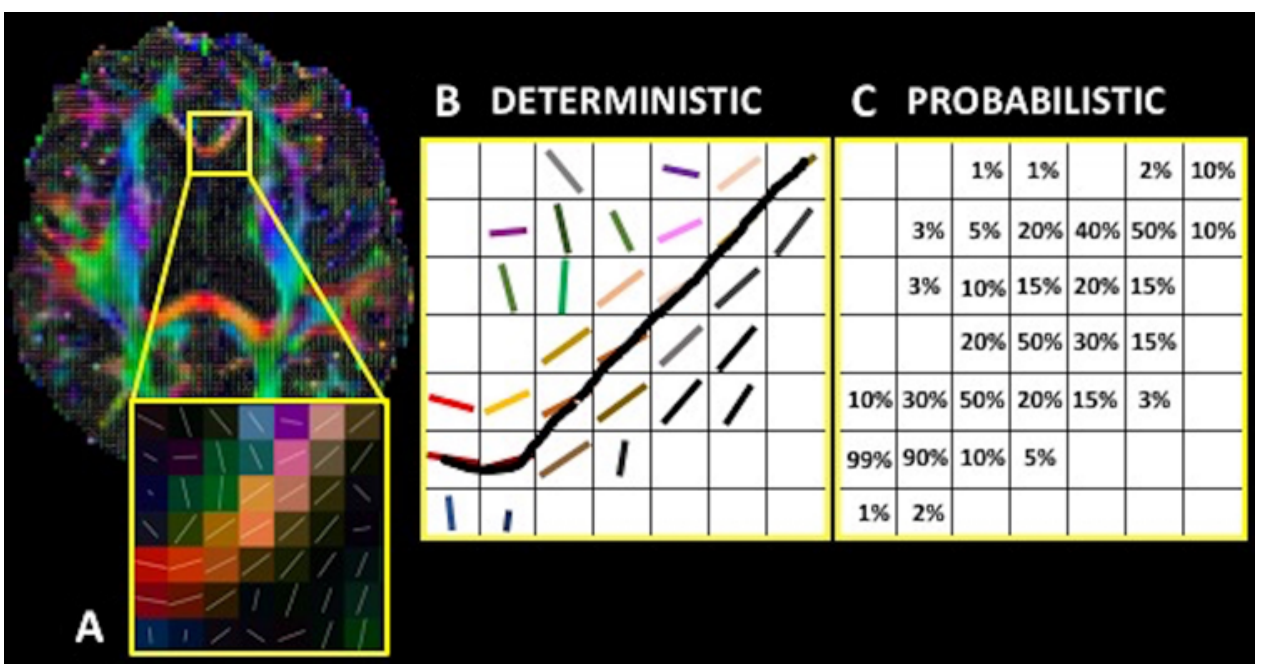

FIG. 1. A: Degree of anisotropy. B: Deterministic fiber tracking; the fiber path across voxels is inferred based on a single diffusion orientation per each voxel. C: Probabilistic fiber tracking; tractography generated based on a probability distribution of diffusion orientations within each voxel.

aminations for surgical planning consisting of T1-weighting scans (with and without gadolinium), T2-weighted scans, and proton density-weighted images; diffusion imaging sequences were also acquired for tractography. All acquisitions were performed on a 3.0-T Philips Achieva MR machine with a 32-channel head coil. To eliminate movement artifact, all patients were scanned under a standardized general anesthetic.

T1-weighted structural scans based on magnetizationprepared rapid acquisition gradient echo (MPRAGE) sequences were acquired for trajectory planning. The T1weighted imaging parameters used were: TR $7.0 \mathrm{msec}$, TE $3.0 \mathrm{msec}$, flip angle $9^{\circ}$, matrix size $512 \times 512$, FOV 25 $\mathrm{cm}$, slice thickness $1.0 \mathrm{~mm}$, and voxel size $1.0 \times 1.0 \times 1.0$ $\mathrm{mm}^{3}$. The T2-weighted and proton density-weighted images were acquired for determining the targets of DBS. A dual spin echo sequence was acquired with the following parameters: TR 5.24 seconds, $\mathrm{TE}_{1} 16 \mathrm{msec}, \mathrm{TE}_{2} 80 \mathrm{msec}$, matrix size $256 \times 256$, slice thickness $2.0 \mathrm{~mm}$, spatial resolution $2.0 \times 2.0 \times 2.0 \mathrm{~mm}^{3}$, and FOV $24 \mathrm{~cm}$. Diffusionweighted images were acquired axially in the same anatomical location prescribed for the T1-weighted images. The following parameters were used: 32 diffusion-encoding directions, $\mathrm{b}=850 \mathrm{sec} / \mathrm{mm}^{2}$, TR 12,000 msec, TE 83 msec, matrix size $128 \times 128$, slice thickness $2.0 \mathrm{~mm}$, and voxel size $1.8 \times 1.8 \times 2.0 \mathrm{~mm}^{3}$. The average acquisition time was 15 minutes for diffusion-weighted images, with a total scan time of approximately 50 minutes.

\section{Manual Segmentation}

For each patient, the GPi and STN were manually segmented using ITK-SNAP (http://www.itksnap.org) by the senior author (C.W.) on the T2-weighted and proton density-weighted scans, respectively. The FreeSurfer analysis suite (http://surfer.nmr.mgh.harvard.edu) was used to automatically parcellate cortical regions of T1-weighted images based on patient anatomy (see Fig. 3, T1-weighted data). Supervision of FreeSurfer segmentation by the se- nior author (C.W.) was performed for the cortical segments of each patient to generate accurate patient-specific parcellation of the motor, supplementary motor, and sensory areas. The GPi, STN, and cortical regions of interest (ROIs) were coregistered to the b0 image obtained from the diffusion scan using the FLIRT (the Functional Magnetic Resonance Imaging of the Brain [FMRIB] Linear Image Registration Tool) rigid body technique with 6 degrees of freedom and nearest-neighbor method as a cost function. The volumes of all segmented ROIs and cortical seeds are shown in Table 2.

\section{Diffusion Data Processing and Tractography}

Diffusion images were corrected for eddy current distortion and motion-induced artifacts using the artefact correction in diffusion MRI (ACID) toolbox (http://www. diffusiontools.com/). Fiber assignment was performed using the selected cortical areas as initiation seeds and the GPi and STN as an inclusion mask. ROIs were manually drawn over the cerebral peduncles of each individual subject's b0 image, and used as an exclusion mask, to ensure corticospinal tracts were not included.

TABLE 1. Patient information for response to DBS and disease severity studies

\begin{tabular}{lcc}
\hline \multicolumn{1}{c}{ Variable } & Mean \pm SD & Range \\
\hline Age (yrs) & $70.4 \pm 7.2$ & $59.1-78.8$ \\
\hline Disease duration (yrs) & $8.2 \pm 2.1$ & $5.7-10.6$ \\
\hline UPDRS-III* & \\
\hline Preop & $29.6 \pm 13.9$ & $15-48$ \\
\hline Postop & $15.8 \pm 4.9$ & $11-23$ \\
\hline Follow-up (mos) & $3.5 \pm 2.5$ & $1.1-7.3$ \\
\hline
\end{tabular}

There were 3 women and 4 men in the study.

* Preoperative medication-ON UPDRS-III and postoperative medication-ON DBS-ON UPDRS-III scores were recorded. 
TABLE 2. Volume of STN, GPi, and cortical seeds

\begin{tabular}{|c|c|c|c|c|c|}
\hline \multirow[b]{2}{*}{ Case No. } & \multicolumn{2}{|c|}{$\operatorname{STN}\left(\mathrm{cm}^{3}\right)$} & \multicolumn{2}{|c|}{$\mathrm{GPi}\left(\mathrm{cm}^{3}\right)$} & \multirow{2}{*}{$\begin{array}{c}\text { Cortical Seeds } \\
\qquad\left(\mathrm{cm}^{3}\right)\end{array}$} \\
\hline & $\mathrm{Lt}$ & $\mathrm{Rt}$ & $\mathrm{Lt}$ & Rt & \\
\hline 1 & 0.16 & 0.14 & 0.44 & 0.66 & 3.34 \\
\hline 2 & 0.18 & 0.15 & 0.46 & 0.43 & 2.33 \\
\hline 3 & 0.16 & 0.16 & 0.52 & 0.54 & 2.17 \\
\hline 4 & 0.13 & 0.15 & 0.59 & 0.42 & 2.79 \\
\hline 5 & 0.15 & 0.15 & 0.51 & 0.44 & 3.54 \\
\hline 6 & 0.16 & 0.15 & 0.51 & 0.45 & 2.87 \\
\hline 7 & 0.15 & 0.16 & 0.53 & 0.49 & 1.90 \\
\hline Mean \pm SD & $0.16 \pm 0.015$ & $0.15 \pm 0.007$ & $0.51 \pm 0.049$ & $0.49 \pm 0.086$ & $2.71 \pm 0.61$ \\
\hline
\end{tabular}

The MRtrix toolbox (https://github.com/jdtournier/ mrtrix3) was used to analyze the diffusion sensitized data set, generate fiber orientation distribution (FOD) estimates, and create deterministic-based track density maps using a CSD model. Probabilistic fiber tractography was performed by the Diffusion Toolbox (FDT) of the FMRIB Software Library. Distributions of fiber orientation were estimated for each voxel based on the BEDPOSTX tool of FDT, computing two crossing fibers per voxel.

Deterministic track density maps and probabilistic maps of sensorimotor regions of the left and right STN and GPi were created. The number of tracts passing through a given voxel were counted and mapped as a numerical value for both tracking methods. Using the manual parcellations of the basal ganglia structures, we created track density images to represent the number of sensorimotor pathways passing through a given ROI.

\section{Track Density Analysis}

In order to compare deterministic and probabilistic tracking solutions, the center of gravity $(\mathrm{CoG})$ of the track density maps of both targeted regions (STN and GPi) was calculated for each patient. For deterministic tractography, the track density map was generated based on whole-brain streamline tractography using FOD (Fig. 2). Within the targeted regions of the STN and GPi, the weighted pixel intensities of the track density maps were multiplied by their corresponding coordinates. The sum was then divided by the total pixel intensity of the track density image of each ROI to find the gray level-weighted CoG. The Euclidean distance was measured between the CoG of deterministic and probabilistic methods. Additionally, to compare the relative location of fiber tracts across each patient in the cohort, the distance between each tracking solution $\mathrm{CoG}$ and the centroid of the target nucleus itself was measured, with the latter serving as a reference point across patients. The standard deviation was calculated across the $x-y-z$ axes for the entire cohort. A dice coefficient statistic was used to compare the sensorimotor regions as defined by each tracking solution. The full processing pipeline is shown in Fig. 3.

\section{Results}

\section{Processing Time}

The estimated processing time for probabilistic fiber tracking ranged from 8 to 10 hours, whereas total processing time for deterministic fiber tracking was estimated to take approximately 5 minutes on a Linux machine with 4 Intel Xeon E5-2697 Processors.

\section{Delineation of the Sensorimotor Regions of the STN and GPi}

Deterministic and probabilistic methods successfully reconstructed pathways between the sensorimotor cortex and the ipsilateral STN (left and right) across all patients. Both methods were able to detect pathways between sensorimotor areas and the ipsilateral GPi (left and right) for all patients. The probabilistic method detected an average of $200 \%$ more pathways connecting the cortical areas to the targeted regions, throughout all structures. Both tracking solutions defined the sensorimotor region within the posterosuperolateral aspect of the ipsilateral STN, and within the posterolateral aspect of the GPi (Fig. 4).

\section{Track Density of Target Regions}

The overlap between the probabilistic and deterministic track density maps within the defined target regions varied considerably between patients; however, the probabilistic method detected a significantly larger portion of fibers for the STN and GPi when compared to the deterministic method, indicating greater sensitivity of the probabilistic method. Overall, the average difference between probabilistic and deterministic tractography $\mathrm{CoG}$ was $2.67 \mathrm{~mm}$. These values are certainly on a relevant order of magnitude for stereotactic trajectory planning. According to the Schaltenbrand-Wahren brain atlas, both the STN and GPi are approximately $4-5 \mathrm{~mm}$ in width. ${ }^{37}$ As such, this discrepancy can mean the difference between targeting the lateral or medial portion of the nucleus. In the realm of DBS, a target difference of $2 \mathrm{~mm}$ can certainly make a clinical difference, particularly if the electrode is implanted closer to the structures that will result in stimulation-related side effects. ${ }^{33}$ Intraoperatively, trajectories are typically adjusted by $2 \mathrm{~mm}$, depending on microelectrode recordings or intraoperative findings.

Both tracking solutions detected the tract $\mathrm{CoG}$ in the posterior and lateral portion of the target nucleus (Table 3). Specifically, the deterministic method yielded a tract CoG 0.17-2.09 mm lateral to the target centroid, which was more medial than the probabilistic derived targets, 

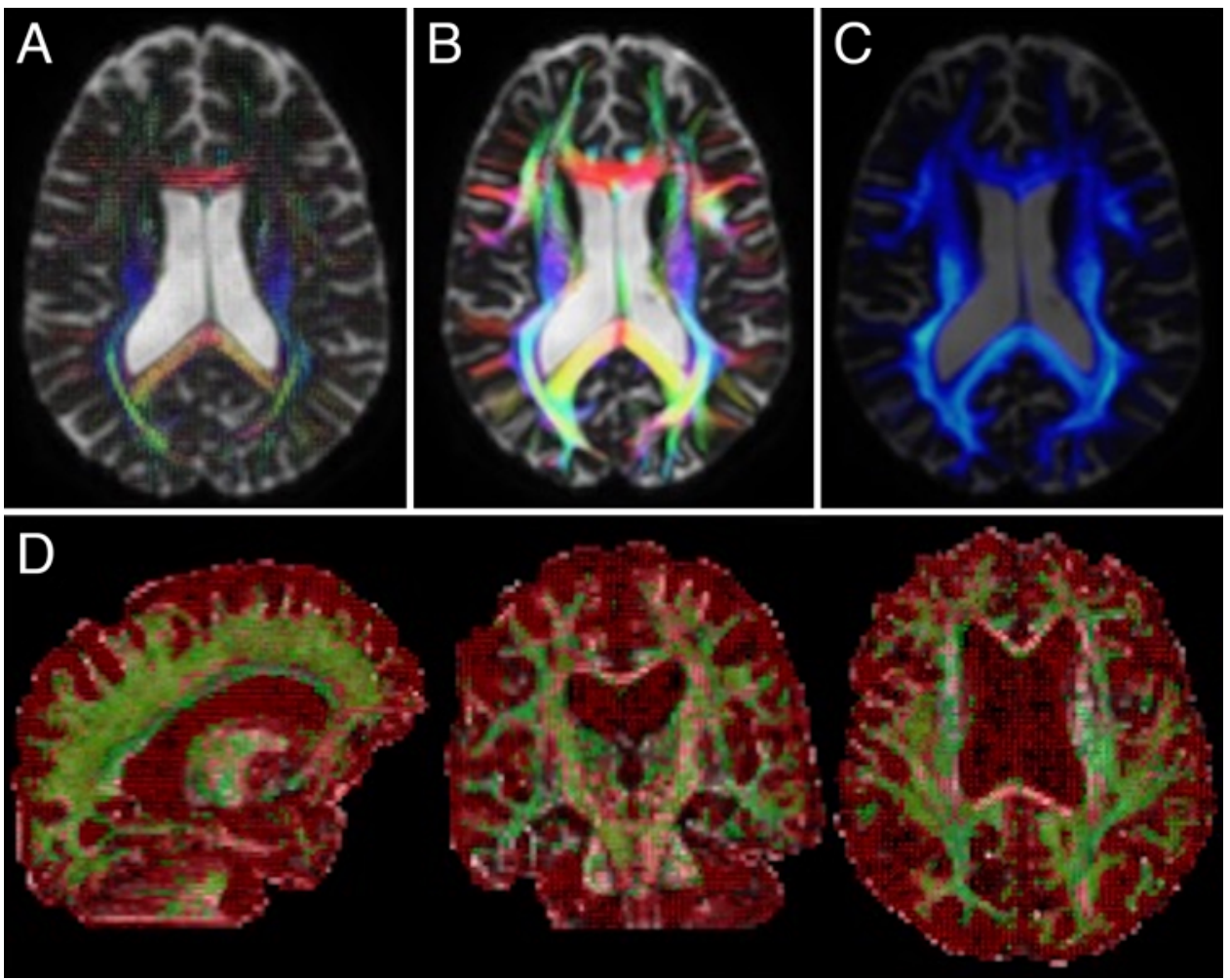

FIG. 2. Extraction of deterministic track density map: FOD estimation performed by CSD technique (A), whole-brain streamline tractography using FOD (B), and track density map based on the streamline tractogram (C). Probabilistic map seeded in the sensorimotor areas; each voxel has a value representing the connectivity value between that voxel and the seed voxel; crossing fibers and fiber orientation are indicated by green and red lines (D).

ranging from 0.53 to $4.09 \mathrm{~mm}$ lateral. This difference was also seen in the y-direction, with deterministic tracking solutions detecting the tract $\mathrm{CoG}$ more anteriorly, ranging between 0.13 and $0.87 \mathrm{~mm}$, while the probabilistic method detected fibers relatively more posteriorly within the nucleus, located between 0.61 and $2.27 \mathrm{~mm}$ from its centroid. Finally, for the z-direction, probabilistic tracking detected more fibers located superiorly within the STN, and slightly inferiorly in the left GPi. Deterministic tracking lacked sensitivity to detect differences between solutions and the centroid of the nucleus in the z-direction for all examined structures.

\section{Discussion}

In this study, the sensorimotor regions of the STN and GPi in patients selected for DBS were delineated based on deterministic and probabilistic fiber tracking techniques. These results, in part, replicate previous work done by Petersen et al., where both tractography methods were used to identify connections between the ipsilateral cortical motor areas to only the STN. ${ }^{32}$ In addition to inclusion of the $\mathrm{GPi}$, a larger patient cohort and a more feasible acquisition time was used in the present study, which increases the clinical applicability for trajectory planning in centers that use either nucleus (STN and/or GPi) as a target for DBS.

\section{Pathophysiology of PD}

Although a detailed review of the literature regarding the pathophysiology of PD is beyond the scope of this study, it is instructive to review the role that the GPi and STN are thought to play in this disease, particularly if we are to better understand the white matter structures being visualized with tractography. Findings suggest that neurons of the internal segment of the GPi and the STN are hyperactive in PD and may contain pathways which, when imbalanced, abnormally activate output nuclei and inhibit thalamic neurons projecting to the cortex. ${ }^{20}$ Cortically initiated activity from voluntary movement is suspected to be regulated by a dichotomy of direct and indirect pathways from the striatum to major output structures of the basal ganglia. ${ }^{9}$ Output neurons of the direct pathway include the substantia nigra pars reticulata and GPi, which are responsible for an increase in locomotor activity and movement. The indirect pathway causes a reduction of locomotor activity and movement through the disinhibition of glutamatergic neurons of the STN. ${ }^{3}$ In this model, PD may be explained as an imbalance between the direct and indirect pathway transmission from the striatum to the basal ganglia output nuclei. ${ }^{20}$

Excitatory projections to the STN from several cortical areas have been termed the "hyperdirect pathway." ${ }^{28}$ This corticosubthalamopallidal hyperdirect pathway conveys excitatory effects from motor-related cortical areas to the globus pallidus, bypassing the striatum, which decreases conduction time for signal propagation. ${ }^{20}$ The hyperdirect pathway is thought to be responsible for the mediation of inhibition that occurs shortly before the time of reaction 


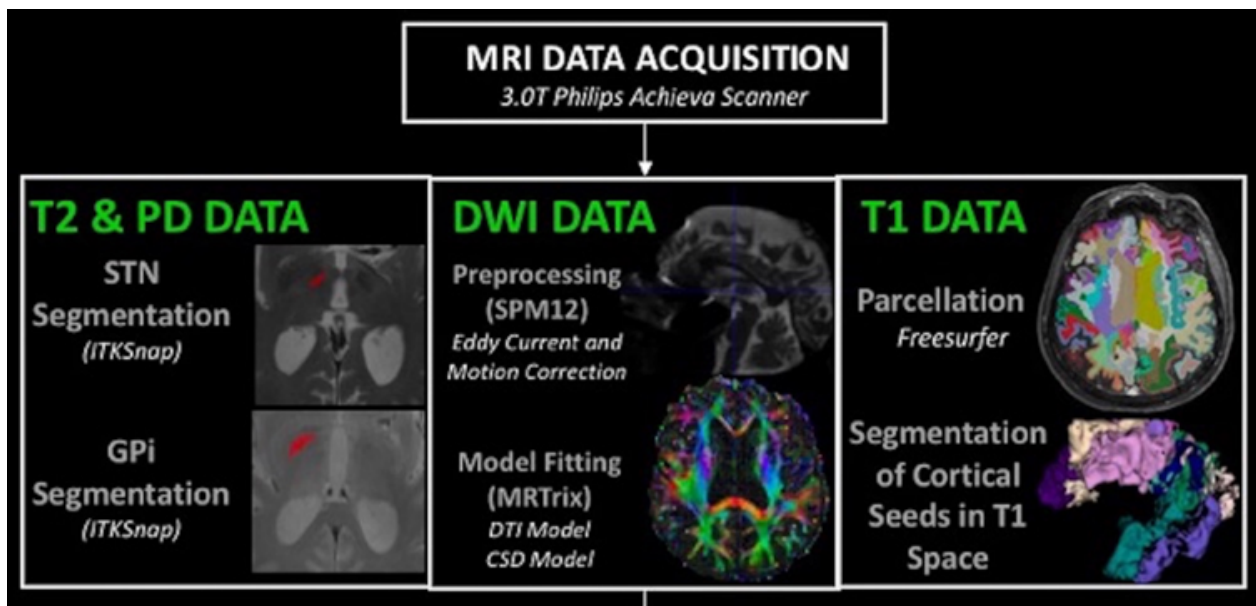

1

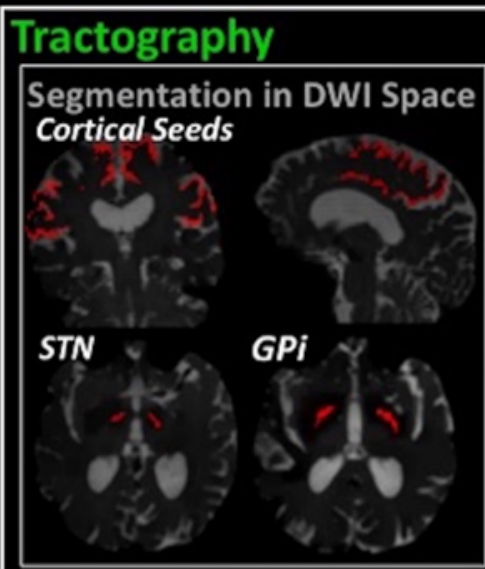

Deterministic Tracking
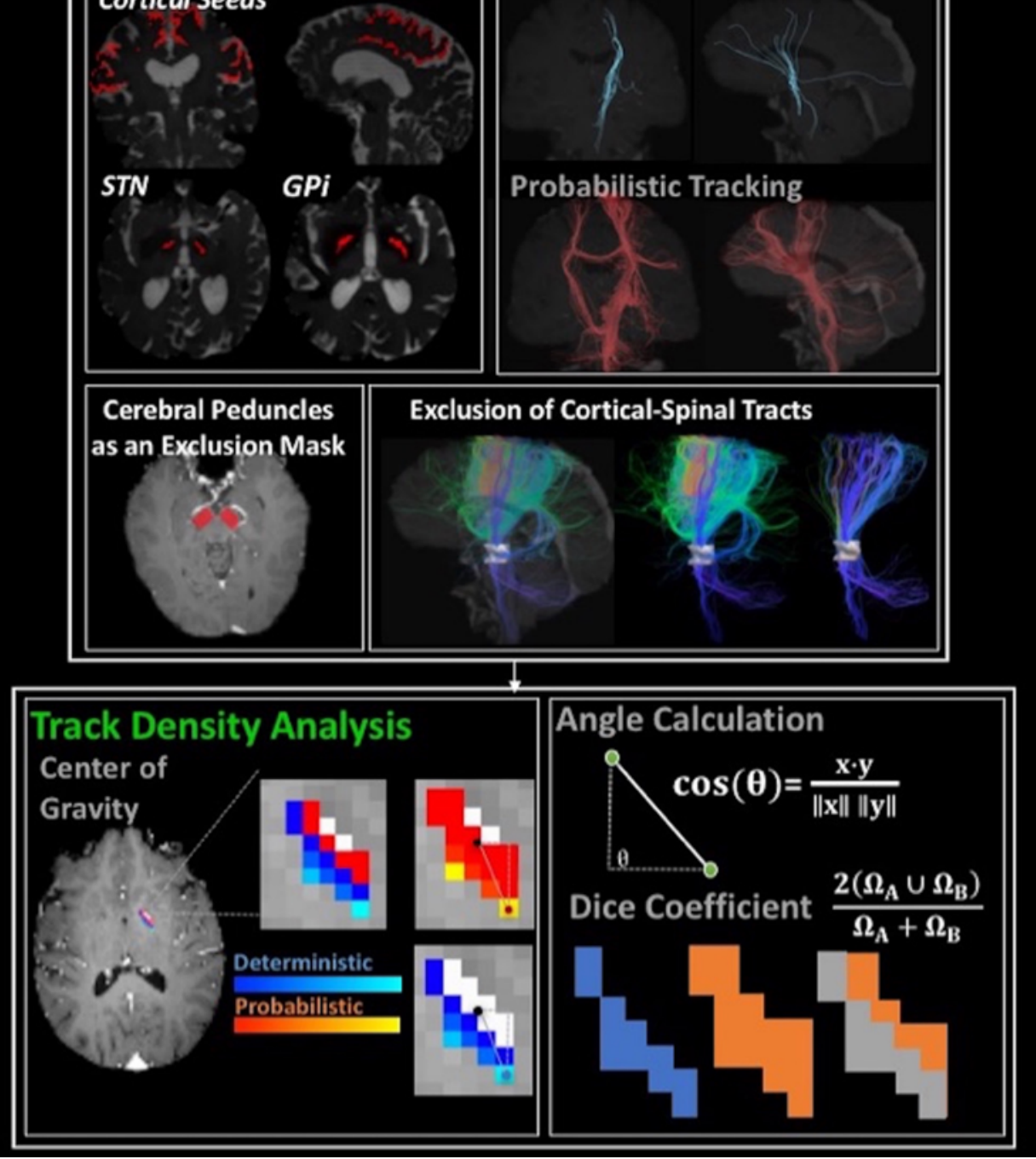

FIG. 3. The full processing pipeline: preprocessing, segmentation, tractogram extraction, and analysis. 

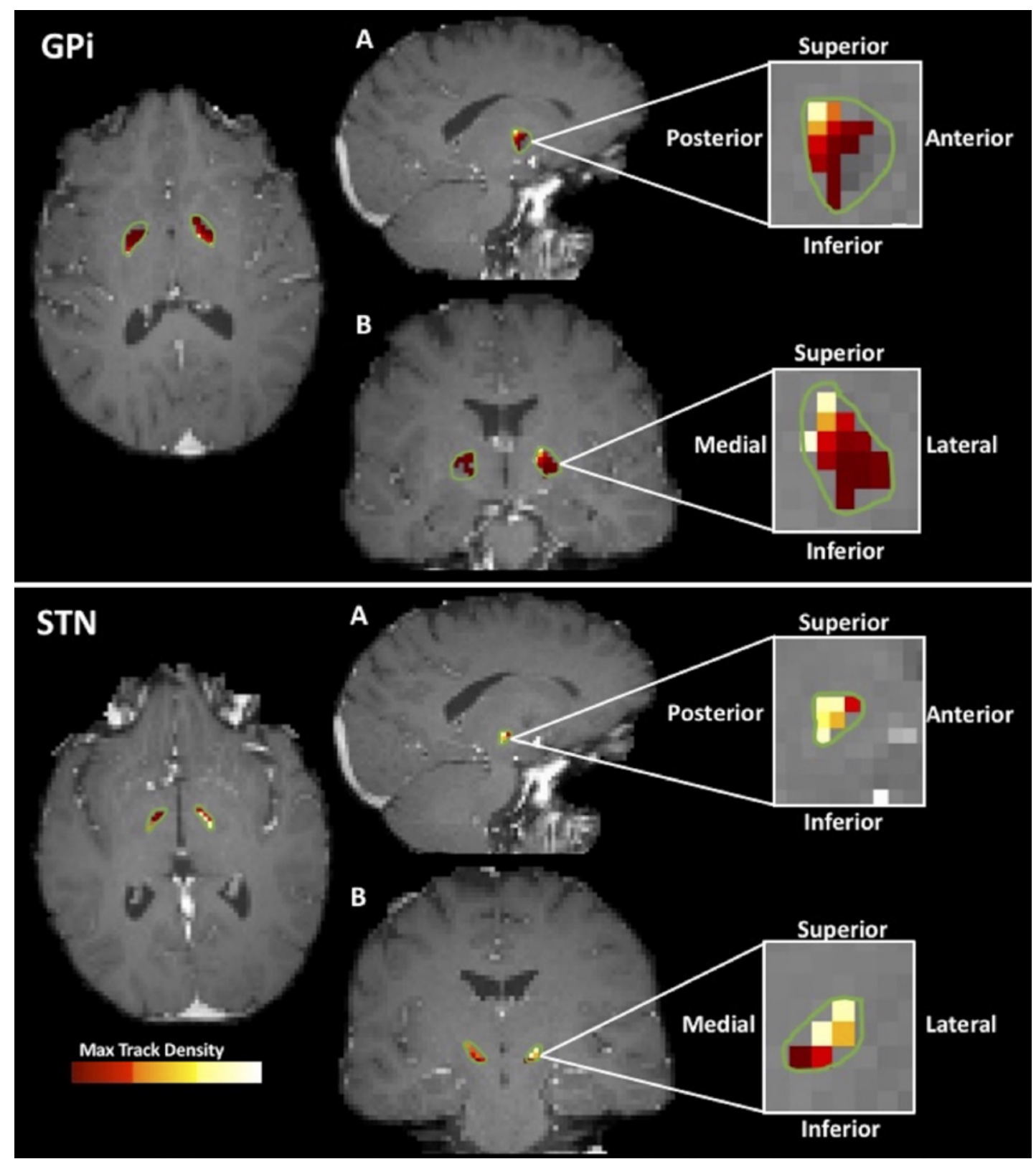

FIG. 4. Anatomical location of track density of probabilistic tractography in the left and right STN and GPI in coronal (A) and saggital (B) views. Maximum fiber density was detected in the posterosuperolateral aspect of the ipsilateral STN and the posterolateral aspect of the GPi.

to external stimuli. ${ }^{12,27,38}$ It is suggested that the hyperdirect pathway can broadly inhibit motor programs so that signals through the direct pathway may be able to adjust the selected motor program. ${ }^{2,12}$ It is because of these relationships that motor portions of the GPi or STN are most commonly targeted in the surgical treatment of PD. . $^{30,34,39,41}$

The direct pathway projects directly to the GPi, whereas the indirect pathway projects first to the globus pallidus externus, which then connects to both the GPi and STN. The hyperdirect pathway is unique as it bypasses the striatum and connects the cortex directly to the STN, which then sends excitatory projections to the GPi.

\section{Detection of Sensorimotor Regions in the GPi and STN and Exclusion of Corticospinal Tracts}

One of the major goals of this work was to detect the location of pathways passing through the GPi and STN with the intent to improve presurgical trajectory planning. By setting the cerebral peduncles as an exclusion mask, all pathways originating in the sensorimotor regions and terminating in the spinal cord or brainstem were eliminated.

We have been careful not to distinguish connections to the GPi as either direct, indirect, or hyperdirect connections, as it is uncertain whether or not the tracts identified first pass through other regions of the fronto-striato-sub- 
thalamic-pallidal network. Both probabilistic and deterministic techniques appeared to connect the sensorimotor cortices with the posterolateral aspect of the GPi and the posterosuperolateral aspect of the ipsilateral STN. This is in accordance with work by Ewert et al., where the GPi and STN were segmented into sensorimotor, associative, and limbic functional zones in non-PD patients, based off of diffusion-based tractography. ${ }^{13}$ Similarly, it has been found that grouped motor, premotor, and sensory areas filled the posterior third of the GPi, which has been described previously as the sensorimotor domain and is a common target for DBS. ${ }^{5,10}$ The posterosuperolateral region of the STN is analogous and has been identified as the sensorimotor functional zone. ${ }^{7,26}$

\section{Probabilistic Versus Deterministic Tracking}

Across all subjects and for all target regions, deterministic fiber tracking reconstructed smaller and more variable streamlines when compared with probabilistic tracking. This variability is clearly appreciated when examining the dice coefficient values (Table 4) for the overlap between track density maps of the GPi, being as low as 0.40 overlap. Furthermore, the Euclidean distance between the two tracking solutions averaged $2.08 \mathrm{~mm}$ for the GPi. While less variability was observed in the confined target region of the STN, a difference between probabilistic and deterministic was still observed, averaging $1.67 \mathrm{~mm}$. This finding implies that the differences between the two tracking methods are still relevant even when considering a smaller target region. Overall, the average difference between probabilistic and deterministic tractography CoG was $2.67 \mathrm{~mm}$. These values are certainly on a relevant order of magnitude for stereotactic trajectory planning. According to the Schaltenbrand-Wahren brain atlas, both the STN and GPi are approximately $4-5 \mathrm{~mm}$ in width. ${ }^{37}$ As such, this difference can mean the difference between targeting the lateral or medial portion of the nucleus. In the realm of DBS, a target difference of $2 \mathrm{~mm}$ can certainly make a clinical difference, particularly if the electrode is implanted closer to structures that will result in stimulation-related side effects. Intraoperatively, trajectories are typically adjusted by $2 \mathrm{~mm}$, depending on microelectrode recordings or intraoperative imaging.

When comparing the distances between the centroid of the targeted nucleus and the CoG of the tracking solutions, a consistently higher standard deviation was observed from the CoG for deterministic tracking, suggesting both higher sensitivity and precision of the probabilistic method. Similar to the work by Petersen et al., ${ }^{32}$ our results demonstrate probabilistic tracking to be a consistently more reliable and accurate method for presurgical trajectory planning.

While our results delineated sensorimotor regions in the posterosuperolateral portion of the STN and posterolateral portion of the GPi (Fig. 3), in 1 patient the difference between probabilistic and deterministic tracking was significantly larger in the left GPi $(13.85 \mathrm{~mm})$ compared to other subjects $(0.16-4.31 \mathrm{~mm})$. Given the abnormal concentration of the tracts in the anterior portion of the GPi produced by the deterministic method, it is likely that this illustrates an incorrect representation of the sensorimotor region. This outlier (Fig. 5, yellow arrow) further empha-
TABLE 3. Coordinate deviation of tracking solutions from the CoG for each of the segmented anatomical nuclei averaged across all patients

\begin{tabular}{ccccc}
\hline Method & $\mathrm{x}(\mathrm{mm})^{*}$ & $\mathrm{y}(\mathrm{mm}) \dagger$ & $\mathrm{z}(\mathrm{mm}) \ddagger$ & Angle $\left(^{\circ}\right)$ \\
\hline Lt STN & & & & \\
\hline Deterministic & -0.34 & -0.25 & 0.00 & 0.25 \\
\hline Probabilistic & -0.53 & -0.61 & 0.43 & 0.36 \\
\hline Rt STN & & & & \\
\hline Deterministic & -0.71 & -0.84 & 0.00 & 0.24 \\
\hline Probabilistic & -0.78 & -1.09 & 0.43 & 0.31 \\
\hline Lt GPi & & & & \\
\hline Deterministic & -2.18 & -0.34 & 0.00 & 0.83 \\
\hline Probabilistic & -3.18 & -1.74 & -0.14 & 2.15 \\
\hline Rt GPi & & & & \\
\hline Deterministic & -4.17 & -1.75 & 0.00 & 0.67 \\
\hline Probabilistic & -4.09 & -2.27 & 0.00 & 0.66 \\
\hline
\end{tabular}

The angle represents the angle of the Euclidian distance vector calculated between the deterministic and probabilistic CoG, and the CoG of the segmented anatomical nucleus, defined in image space.

* $+=$ anterior; $-=$ posterior.

$\dagger+=$ medial; $-=$ lateral.

$\ddagger+=$ superior; - = inferior.

sizes the limitations of deterministic tractography and the increased accuracy of its probabilistic counterpart.

Ultimately, targeting the motor subregion of the nucleus has been shown to yield improved motor outcomes in DBS for PD. ${ }^{4}$ As such, we believe that the differences between deterministic and probabilistic tracking methods are significant enough to warrant the increased processing requirements, particularly because situations may arise where the formed tractography yields unusable results.

\section{Clinical Application}

The use of advanced imaging techniques to accurately demonstrate white matter tracts in a $3 \mathrm{D}$ manner is useful in neurosurgery for both decision making and neurosurgical planning. ${ }^{18}$ While a number of studies have demonstrated this utility, the time required for image acquisition often limits the clinical applicability of advanced diffusion imaging methods. As such, we aimed to develop an imaging pipeline capable of accurate white matter tracking while maintaining a short acquisition time. Our diffusionweighted images, with a spatial resolution of $2.0 \times 2.0 \times$ $2.0 \mathrm{~mm}^{3}$ and 32 directions, were acquired in merely 15 minutes. Previous work conducted in reconstructing STN projections using probabilistic tractography have used longer scan times to achieve high-resolution DTI with an acquisition time of approximately 1 hour and 30 minutes. $^{32}$ While a lengthy scan time may be a minor inconvenience in a research setting, the duration of the acquisition would not be feasible in a routine clinical setting. Currently no probabilistic methods of tractography are commercially available packages cleared for clinical use. This work builds the argument for increasing the availability of advanced diffusion methods for use in a clinical setting. 
TABLE 4. Difference in target regions' CoG coordinates between tracking methods

\begin{tabular}{|c|c|c|c|c|}
\hline \multirow[b]{2}{*}{ Case No. } & \multicolumn{2}{|c|}{ CoG Coordinates of Track Density (image space) } & \multirow{2}{*}{$\begin{array}{l}\text { Euclidean Distance }(\mathrm{mm}) \text { Btwn } \\
\text { Both Tracking Solutions* }\end{array}$} & \multirow{2}{*}{$\begin{array}{c}\text { Dice } \\
\text { Coefficient }\end{array}$} \\
\hline & Deterministic & Probabilistic & & \\
\hline \multicolumn{5}{|l|}{1} \\
\hline STN Lt & $62.4,69.4,25.5$ & $62.0,69.6,25.5$ & 1.63 & 1.00 \\
\hline STN Rt & $63.2,57.1,25.5$ & $62.9,56.5,25.5$ & 2.74 & 0.98 \\
\hline GPi Lt & $63.2,74.6,29.5$ & $63.0,75.1,29.5$ & 2.11 & 0.83 \\
\hline GPi Rt & $62.8,50.9,28.0$ & $62.7,50.7,28.0$ & 1.06 & 0.67 \\
\hline \multicolumn{5}{|l|}{2} \\
\hline STN Lt & $55.3,70.5,39.5$ & $55.0,70.6,39.5$ & 1.18 & 1.00 \\
\hline STN Rt & $55.9,57.7,39.0$ & $56.2,57.7,39.5$ & 2.21 & 0.98 \\
\hline GPi Lt & $57.5,74.0,42.5$ & $57.1,75.2,42.5$ & 4.92 & 0.63 \\
\hline GPi Rt & $59.0,54.2,42.5$ & $59.3,53.9,42.5$ & 1.46 & 0.83 \\
\hline \multicolumn{5}{|l|}{3} \\
\hline STN Lt & $63.7,71.1,35.0$ & $63.6,71.1,35.0$ & 0.22 & 1.00 \\
\hline STN Rt & $62.2,58.9,34.5$ & $62.2,58.9,34.5$ & 0.33 & 1.00 \\
\hline GPi Lt & $68.8,72.0,36.5$ & $66.1,74.2,37.0$ & 14.44 & 0.40 \\
\hline GPi Rt & $65.0,56.0,38.0$ & $64.5,55.4,38.0$ & 3.10 & 0.84 \\
\hline \multicolumn{5}{|l|}{4} \\
\hline STN Lt & $59.1,71.0,39.5$ & $58.8,71.0,39.5$ & 0.41 & 0.94 \\
\hline STN Rt & $60.1,58.0,40.5$ & $59.7,57.7,40.5$ & 0.16 & 0.98 \\
\hline GPi Lt & $61.5,75.1,43.0$ & $61.7,75.3,43.0$ & 1.40 & 0.61 \\
\hline GPi Rt & $62.8,54.1,45.5$ & $62.6,53.5,45.0$ & 1.71 & 0.67 \\
\hline \multicolumn{5}{|l|}{5} \\
\hline STN Lt & $65.1,70.0,34.0$ & $63.7,71.3,34.0$ & 2.86 & 0.95 \\
\hline STN Rt & $63.6,57.1,34.5$ & $62.8,56.6,34.5$ & 2.28 & 0.93 \\
\hline GPi Lt & $64.9,74.8,38.5$ & $64.6,75.2,38.5$ & 2.34 & 0.86 \\
\hline GPi Rt & $65.8,54.1,40.0$ & $64.9,52.8,39.5$ & 2.39 & 0.76 \\
\hline \multicolumn{5}{|l|}{6} \\
\hline STN Lt & $62.4,70.3,38.5$ & $62.8,70.6,39.0$ & 2.64 & 0.97 \\
\hline STN Rt & $62.5,57.0,39.0$ & $62.9,57.1,39.0$ & 1.52 & 1.00 \\
\hline GPi Lt & $64.8,74.1,41.0$ & $64.9,74.1,41.0$ & 0.20 & 0.71 \\
\hline GPi Rt & $63.7,52.5,40.5$ & $64.7,52.9,41.0$ & 4.61 & 0.68 \\
\hline \multicolumn{5}{|l|}{7} \\
\hline STN Lt & $64.6,68.6,40.0$ & $64.3,68.9,40.0$ & 1.72 & 1.00 \\
\hline STN Rt & $64.7,57.3,41.0$ & $64.7,57.3,41.0$ & 0.16 & 1.00 \\
\hline GPi Lt & $67.9,70.7,44.5$ & $68.0,71.0,44.5$ & 1.48 & 0.75 \\
\hline GPi Rt & $65.2,53.5,44.0$ & $65.1,53.4,44.0$ & 0.65 & 0.73 \\
\hline
\end{tabular}

All coordinates have been reported in image space with the diffusion image serving as the reference. A dice coefficient of 1 indicates a perfect overlap, whereas a dice coefficient of 0 indicates no overlap.

* The GPi median ( \pm SD) was $2.08 \pm 3.69 \mathrm{~mm}$, whereas the STN median was $1.67 \pm 1.90 \mathrm{~mm}$. The total average distance was $2.67 \mathrm{~mm}$.

\section{Limitations and Future Work}

Although a shorter acquisition time is desirable for clinical feasibility, the resulting low resolution of our DTI data creates a limitation when performing sensitive and accurate fiber tractography. The processing time for the creation of the track density maps, consisting of both manual and automated segmentation, FOD estimation, and whole-brain tractography, is associated with a substantial expenditure of time, which can be inefficient when working within a clinical timeframe. Probabilistic tracking required up to 10 hours of processing time on a typical computer processor. Such processing could be incorporated into the workflows of surgeons who routinely plan their trajectories prior to the day of surgery, but would not be able to accommodate those who routinely perform stereotactic planning immediately prior to the surgical procedure. That being said, in their identification of the subthalamic region stimulation clusters, Akram et al. were able to successfully delineate fiber orientations using a graphics processing unit (GPU). ${ }^{2}$ In the future, 

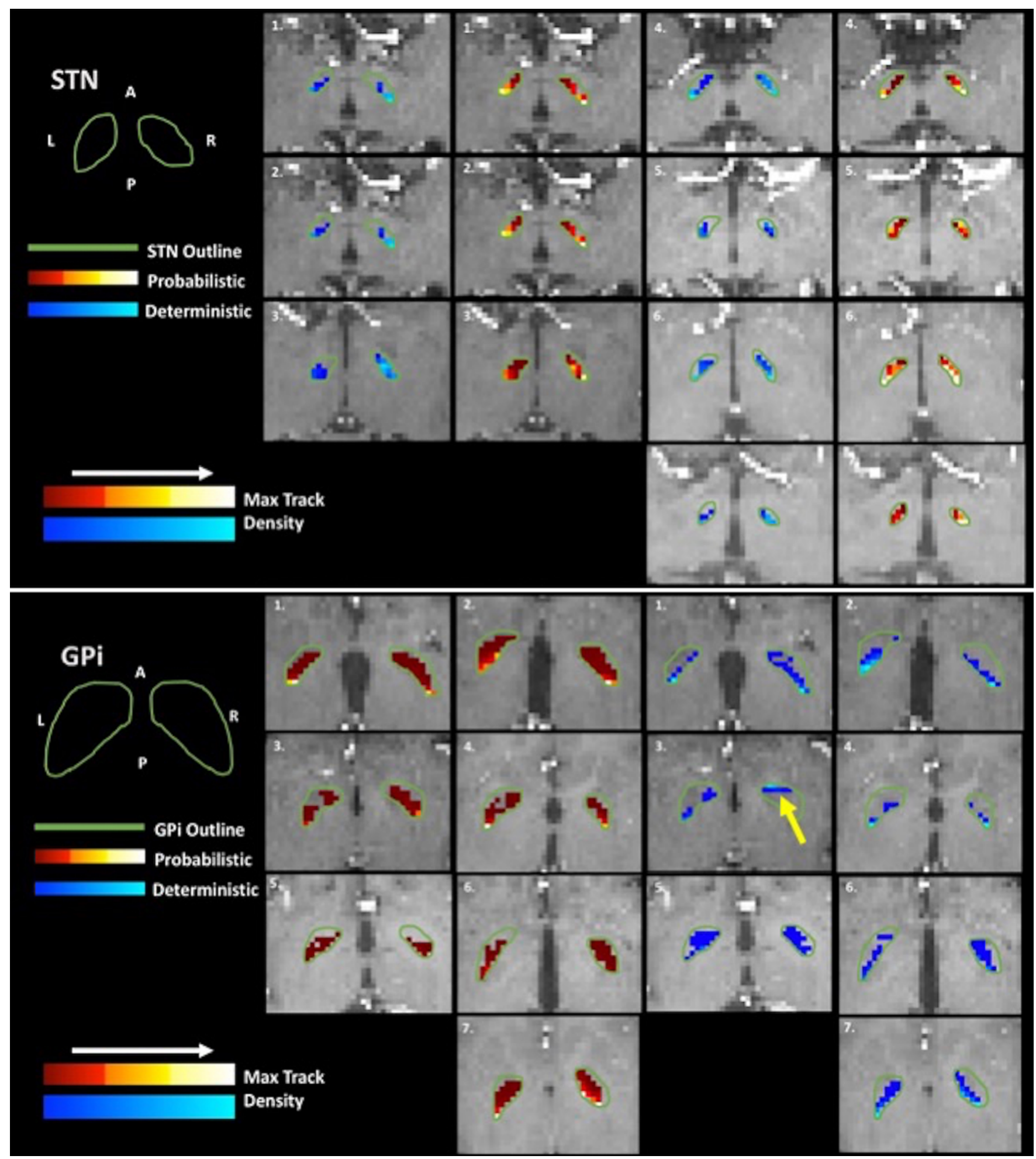

FIG. 5. Cross-sectional view of probabilistic and deterministic track density maps of direct cortico-GPi pathways within the STN and GPi (left and right). The sensorimotor region is most concentrated in the posterosuperolateral portion of the STN, and the posterolateral portion of the GPi. Note that in case 3 (yellow arrow) the deterministic method inaccurately detected the CoG to be anterior in the GPi. 
technological advances in processing capabilities such as GPU processing will help shorten processing times and consequently allow for greater adoption of these tracking techniques in the preoperative clinical setting. GPU-based data processing hardware is becoming more available in radiology settings. ${ }^{18}$ In either scenario, however, personnel with expertise in probabilistic tractography would be needed to process diffusion data accordingly.

A significant amount of research remains to be undertaken for the clinical use of tractography-based DBS targeting. First, the current study is limited by its small sample size. Second, to better understand the clinical relevance of tractography-based DBS targeting, clinical outcomes must also be considered. We intend to analyze the relationship between track density maps and electrode placement to quantify the effect of electrode placement on clinical outcomes. This will involve the calculation of the volume of tissue activation, calculated from the programming parameters, so as not to overlook the effect contacts adjacent to the nucleus might have on cell bodies as well as axons within the white matter outside of the nucleus. ${ }^{2}$ The aim of this future study will be to associate overlapping stimulation sites within the STN and GPi with different connectivity patterns for maximum improvement in UPDRS-III scores.

We hope that our efforts will allow us to better understand the anatomical relationships and networks in PD. Because tractography illustrates connections without providing insight into the directionality, or number of connections between ROIs, it is possible that a portion of the fibers detected individually in the GPi and STN track density maps may pass through additional structures. In the future the use of additional inclusion and exclusion regions after performing tractography may allow for successful delineation of the direct, indirect, and hyperdirect pathways. By doing so, a better understanding of these three pathways and their contribution to disease progression and severity may be gleaned.

\section{Conclusions}

The results of this study demonstrate probabilistic tractography as a more reliable tractography method when compared to the deterministic-based method, in both the regions of the STN and GPi. Analysis of the results of probabilistic and deterministic tractography for both the STN and GPi indicates that probabilistic tracking is a more robust method for accurate stereotactic presurgical planning. Furthermore, we have demonstrated the ability to detect sensorimotor regions of the GPi and STN within a clinically applicable timeframe, presenting the opportunity for the integration of advanced neuroimaging methods for presurgical planning.

\section{References}

1. Abhinav K, Yeh FC, Pathak S, Suski V, Lacomis D, Friedlander RM, et al: Advanced diffusion MRI fiber tracking in neurosurgical and neurodegenerative disorders and neuroanatomical studies: a review. Biochim Biophys Acta 1842:2286-2297, 2014

2. Akram H, Sotiropoulos SN, Jbabdi S, Georgiev D, Mahlknecht P, Hyam J, et al: Subthalamic deep brain stimulation sweet spots and hyperdirect cortical connectivity in Parkinson's disease. Neuroimage 158:332-345, 2017
3. Aron AR, Poldrack RA: Cortical and subcortical contributions to Stop signal response inhibition: role of the subthalamic nucleus. J Neurosci 26:2424-2433, 2006

4. Aviles-Olmos I, Kefalopoulou Z, Tripoliti E, Candelario J, Akram H, Martinez-Torres I, et al: Long-term outcome of subthalamic nucleus deep brain stimulation for Parkinson's disease using an MRI-guided and MRI-verified approach. J Neurol Neurosurg Psychiatry 85:1419-1425, 2014

5. Baker KB, Lee JY, Mavinkurve G, Russo GS, Walter B, DeLong MR, et al: Somatotopic organization in the internal segment of the globus pallidus in Parkinson's disease. Exp Neurol 222:219-225, 2010

6. Behrens TEJ, Johansen-Berg H, Woolrich MW, Smith SM, Wheeler-Kingshott CAM, Boulby PA, et al: Non-invasive mapping of connections between human thalamus and cortex using diffusion imaging. Nat Neurosci 6:750-757, 2003

7. Bejjani BP, Dormont D, Pidoux B, Yelnik J, Damier P, Arnulf I, et al: Bilateral subthalamic stimulation for Parkinson's disease by using three-dimensional stereotactic magnetic resonance imaging and electrophysiological guidance. J Neurosurg 92:615-625, 2000

8. Brock M, Kern BC, Funk T, Afshar HF: Pallidal or subthalamic stimulation. J Neurosurg 89:345-346, 1998

9. Chiken S, Nambu A: Mechanism of deep brain stimulation: inhibition, excitation, or disruption? Neuroscientist 22:313322, 2016

10. Cif L, Gonzalez-Martinez V, Vasques X, Corlobé A, Moura AM, Bonafé A, et al: Staged implantation of multiple electrodes in the internal globus pallidus in the treatment of primary generalized dystonia. J Neurosurg 116:1144-1152, 2012

11. Descoteaux M, Deriche R, Knösche TR, Anwander A: Deterministic and probabilistic tractography based on complex fibre orientation distributions. IEEE Trans Med Imaging 28:269-286, 2009

12. Deuschl G, Schade-Brittinger C, Krack P, Volkmann J, Schäfer H, Bötzel K, et al: A randomized trial of deep-brain stimulation for Parkinson's disease. N Engl J Med 355:896-908, 2006

13. Ewert S, Plettig P, Li N, Chakravarty MM, Collins DL, Herrington TM, et al: Toward defining deep brain stimulation targets in MNI space: A subcortical atlas based on multimodal MRI, histology and structural connectivity. Neuroimage 170:271-282, 2018

14. Follett KA, Weaver FM, Stern M, Hur K, Harris CL, Luo P, et al: Pallidal versus subthalamic deep-brain stimulation for Parkinson's disease. N Engl J Med 362:2077-2091, 2010

15. Gunalan K, Chaturvedi A, Howell B, Duchin Y, Lempka SF, Patriat R, et al: Creating and parameterizing patient-specific deep brain stimulation pathway-activation models using the hyperdirect pathway as an example. PLoS One 12:e176132, 2017

16. Guridi J, Rodriguez-Oroz MC, Lozano AM, Moro E, Albanese A, Nuttin B, et al: Targeting the basal ganglia for deep brain stimulation in Parkinson's disease. Neurology 55 (12 Suppl 6):S21-S28, 2000

17. Hariz MI, Rehncrona S, Quinn NP, Speelman JD, Wensing C: Multicenter study on deep brain stimulation in Parkinson's disease: an independent assessment of reported adverse events at 4 years. Mov Disord 23:416-421, 2008

18. Hernández M, Guerrero GD, Cecilia JM, García JM, Inuggi A, Jbabdi S, et al: Accelerating fibre orientation estimation from diffusion weighted magnetic resonance imaging using GPUs. PLoS One 8:e61892, 2013

19. Hesseltine SM, Ge Y, Law M: Applications of diffusion tensor imaging and fiber tractography. Appl Radiol 36:8-23, 2007

20. Jahanshahi M, Obeso I, Rothwell JC, Obeso JA: A frontostriato-subthalamic-pallidal network for goal-directed and habitual inhibition. Nat Rev Neurosci 16:719-732, 2015 
21. Jeurissen B, Leemans A, Tournier JD, Jones DK, Sijbers J: Investigating the prevalence of complex fiber configurations in white matter tissue with diffusion magnetic resonance imaging. Hum Brain Mapp 34:2747-2766, 2013

22. Jones DK, Leemans A: Diffusion tensor imaging. Methods Mol Biol 711:127-144, 2011

23. Krack P, Limousin P, Benabid AL, Pollak P: Chronic stimulation of subthalamic nucleus improves levodopa-induced dyskinesias in Parkinson's disease. Lancet 350:1676, 1997

24. Krause M, Fogel W, Heck A, Hacke W, Bonsanto M, Trenkwalder C, et al: Deep brain stimulation for the treatment of Parkinson's disease: subthalamic nucleus versus globus pallidus internus. J Neurol Neurosurg Psychiatry 70:464-470, 2001

25. Kristo G, Leemans A, Raemaekers M, Rutten GJ, de Gelder B, Ramsey NF: Reliability of two clinically relevant fiber pathways reconstructed with constrained spherical deconvolution. Magn Reson Med 70:1544-1556, 2013

26. Lambert C, Zrinzo L, Nagy Z, Lutti A, Hariz M, Foltynie $\mathrm{T}$, et al: Confirmation of functional zones within the human subthalamic nucleus: patterns of connectivity and subparcellation using diffusion weighted imaging. Neuroimage 60:83-94, 2012

27. Massano J, Garrett C: Deep brain stimulation and cognitive decline in Parkinson's disease: a clinical review. Front Neurol 3:66, 2012

28. Nambu A, Tokuno H, Takada M: Functional significance of the cortico-subthalamo-pallidal 'hyperdirect' pathway. Neurosci Res 43:111-117, 2002

29. Okun MS, Fernandez HH, Wu SS, Kirsch-Darrow L, Bowers $\mathrm{D}$, Bova F, et al: Cognition and mood in Parkinson's disease in subthalamic nucleus versus globus pallidus interna deep brain stimulation: the COMPARE trial. Ann Neurol 65:586595,2009

30. Østergaard K, Aa Sunde N: Evolution of Parkinson's disease during 4 years of bilateral deep brain stimulation of the subthalamic nucleus. Mov Disord 21:624-631, 2006

31. Patriat R, Cooper SE, Duchin Y, Niederer J, Lenglet C, Aman J, et al: Individualized tractography-based parcellation of the globus pallidus pars interna using 7T MRI in movement disorder patients prior to DBS surgery. Neuroimage 178:198-209, 2018

32. Petersen MV, Lund TE, Sunde N, Frandsen J, Rosendal F, Juul N, et al: Probabilistic versus deterministic tractography for delineation of the cortico-subthalamic hyperdirect pathway in patients with Parkinson disease selected for deep brain stimulation. J Neurosurg 126:1657-1668, 2017

33. Richter EO, Hoque T, Halliday W, Lozano AM, Saint-Cyr JA: Determining the position and size of the subthalamic nucleus based on magnetic resonance imaging results in patients with advanced Parkinson disease. J Neurosurg 100:541-546, 2004

34. Rodrigues JP, Walters SE, Watson P, Stell R, Mastaglia FL:
Globus pallidus stimulation improves both motor and nonmotor aspects of quality of life in advanced Parkinson's disease. Mov Disord 22:1866-1870, 2007

35. Rodriguez-Oroz MC, Rodriguez M, Guridi J, Mewes K, Chockkman V, Vitek J, et al: The subthalamic nucleus in Parkinson's disease: somatotopic organization and physiological characteristics. Brain 124:1777-1790, 2001

36. Romanelli P, Bronte-Stewart H, Heit G, Schaal DW, Esposito $\mathrm{V}$ : The functional organization of the sensorimotor region of the subthalamic nucleus. Stereotact Funct Neurosurg 82:222-229, 2004

37. Schaltenbrand G, Wahren W: Atlas for Stereotaxy of the Human Brain. New York: Thieme, 1998.

38. Schlaier JR, Beer AL, Faltermeier R, Fellner C, Steib K, Lange M, et al: Probabilistic vs. deterministic fiber tracking and the influence of different seed regions to delineate cerebellar-thalamic fibers in deep brain stimulation. Eur J Neurosci 45:1623-1633, 2017

39. Smith Y, Wichmann T: The cortico-pallidal projection: an additional route for cortical regulation of the basal ganglia circuitry. Mov Disord 30:293-295, 2015

40. Wider C, Pollo C, Bloch J, Burkhard PR, Vingerhoets FJG: Long-term outcome of 50 consecutive Parkinson's disease patients treated with subthalamic deep brain stimulation. Parkinsonism Relat Disord 14:114-119, 2008

41. Williams NR, Foote KD, Okun MS: STN vs. GPi deep brain stimulation: translating the rematch into clinical practice. Mov Disord Clin Pract (Hoboken) 1:24-35, 2014

\section{Disclosures}

The authors report no conflict of interest concerning the materials or methods used in this study or the findings specified in this paper.

\section{Author Contributions}

Conception and design: Muller, Alizadeh, Mohamed, Trieu, Wu. Acquisition of data: Alizadeh, Mohamed, Riley, Pearce, Liang, Romo, Wu. Analysis and interpretation of data: Muller, Alizadeh, Pearce, Wu. Drafting the article: Muller, Alizadeh, Wu. Critically revising the article: Muller, Alizadeh, Mohamed, Pearce, Trieu, Romo, Wu. Reviewed submitted version of manuscript: all authors. Approved the final version of the manuscript on behalf of all authors: Muller. Statistical analysis: Muller, Alizadeh, Wu. Administrative/technical/material support: Mohamed, Riley, Liang, Sharan, Wu. Study supervision: Mohamed, Riley, Sharan, Wu.

\section{Correspondence}

Jennifer Muller: Thomas Jefferson University, Philadelphia, PA. jenmuller645@gmail.com. 